\title{
Galactic Astronomy: Past, Present \& Future A Personal View
}

\author{
Johannes Andersen ${ }^{1,2}$ \\ ${ }^{1}$ The Niels Bohr Institute, University of Copenhagen, \\ Juliane Maries Vej 30, DK-2100, Copenhagen, Denmark \\ email: ja@nbi.ku.dk \\ ${ }^{2}$ Nordic Optical Telescope, Apartado 474, ES 38700 Santa Cruz de La Palma, Spain \\ email: ja@not.iac.es
}

\begin{abstract}
This closing contribution is structured as a tour of developments of Galactic astronomy over the last forty years, seen from an entirely personal viewpoint. On this background, I try to place some of the current developments in context, with reference to many of the key contributions to this meeting, and speculate where we may be heading in the near future.
\end{abstract}

Keywords. Galaxy: general; Galaxy: evolution

The organisers kindly invited me to present a closing talk with the above title at IAU Symposium 298. I cannot, of course, possibly pretend to do justice to such a grand title during my half hour, and it would be utterly boring for the audience to hear me reeling off the titles of the many interesting talks and posters of the past week in chronological order. With the organisers' permission, I have chosen instead to divide the history of the past forty years into chapters exemplified by the main projects in which I have personally been involved, together with Birgitta Nordström and many other collaborators. On this background, I note the subsequent developments of those fields up to today and beyond, from the perspective of what I believe I learned during this fascinating and informative week. I apologise in advance for any incompleteness or omissions in this brief summary.

\section{Scientific State of the Field Around 1973}

After some years of testing the stellar models and age scales of the epoch with precise light and radial velocity curves of eclipsing binaries, we took the first modest steps into Galactic astronomy around 1973. From the perspective of this week's series of excellent reviews, it is sobering to recall the state of our knowledge of the Galaxy at that time:

- The basic scheme of the Galactic populations had been defined at the Vatican conference in 1958, including the "Intermediate Population II", later to be rediscovered under the name "Thick Disk".

- The Hubble constant - hence the age of the Universe - was only known to within roughly a factor two.

- The existence of dark matter had been demonstrated by Fritz Zwicky, but was not believed by mainstream astronomers.

- The basic spiral structure of the disk was "known" from $21 \mathrm{~cm}$ observations and kinematics of H II regions, but no consistent or agreed picture existed.

- The overall spatial distribution of globular clusters was known. Globular clusters were examples of pure stellar populations, and some were the oldest and most metalpoor objects in the Universe. 
- Determinations of ages and metallicities for small samples of Population I stars existed, but the uncertainties were large.

- Metallicities were known for a few halo stars, but only down to the limit defined by the globular clusters, $[\mathrm{Fe} / \mathrm{H}] \sim-2.3$.

- Reliable distance determinations from trigonometric parallaxes existed only for a few hundred stars, and even the distance of the Hyades moving cluster remained controversial. Proper motions for $\sim 250,000$ stars were available from the SAO catalogue, but the data were compiled from on older photographic surveys.

- The sites and processes of star formation in the Milky way were basically unknown.

- On the observational side, photographic plates and photomultipliers were basically the only available detectors. Classification spectra were available from the HD and other survey catalogues, but extracting precise quantitative information from them (radial velocities, spectrophotometry) was a slow and laborious process.

\section{Programmes in Galactic Structure in Copenhagen 1973 - 1990}

The year 1967 was marked by two events of major importance for Danish astronomy: Denmark joined ESO, and Prof. Bengt Strömgren returned from many years in the USA, bringing a comprehensive and up-to-date understanding of the theoretical and observational tools needed to address fundamental issues in the evolution of our Galaxy, based on his own seminal contributions. At the same time, the outstanding observing conditions in Chile offered unprecedented scope for ambitious survey programmes. These opportunities inspired and enabled our group to undertake a broad attack on key aspects of the problem:

On the theoretical side, stellar interior models were developed and refined (using a computer with a total memory capacity of $1 \mathrm{k}$ 42-bit words!), and Bengt Gustafsson - then a postdoc at NORDITA in Copenhagen - began his work to develop the next generation of stellar atmospheres. New, efficient multichannel instruments for uvby $\beta$ photoelectric photometry were designed and built to determine distances, metallicities and ages for BAFG stars, and a $50 \mathrm{~cm}$ telescope was moved from Denmark to the ESO observatory on La Silla, Chile, to improve its productivity. To someone from the cloudy lowlands of Denmark, the Chilean climate was a revolution: Suddenly large programmes were something one could plan and expect to complete on schedule!

Proper motions were also determined from observations with a new meridian circle, first operated in Denmark with photographic recording, later converted to photoelectric detection, automated, and in 1984 moved to the Roque de los Muchachos Observatory on La Palma, Spain, where it continues to operate entirely autonomously.

But the missing link in the chain was radial velocities, at the time still to be measured from photographic spectra. The Bright Star Catalog was being completed in uvby $\beta$ photometry, but $\sim 1,500$ southern HR stars had no radial-velocity data at all. So Birgitta and I initiated our first "large survey", comprising the early-type half of these stars, and $\sim 3,000$ spectrograms were obtained over 65 nights at the ESO $1.5 \mathrm{~m}$ telescope and measured with an efficient, but still manually operated measuring machine.

In the meantime, a project had been launched to build a modern $1.5 \mathrm{~m}$ Danish telescope on La Silla in cooperation with ESO, and an agreement was reached to install a copy of the Geneva-Marseille photoelectric radial-velocity scanner CORAVEL there. This would greatly speed up the programme to measure radial velocities for the remaining latetype HR and other stars. Technical and managerial problems delayed the telescope - a recurrent theme during all these decades! - but the southern CORAVEL was eventually 
commissioned in 1981 and produced well over 100,000 observations on a large variety of galactic projects over the following 16 years.

Meanwhile, the uvby $\beta$ photometric surveys had been extended by Erik Heyn Olsen to all $\sim 30,000$ AFG stars in the whole sky to $V=8.3$, and the CORAVELs enabled us to gradually expand the radial velocity project to include all the $\sim 14,000$ Solar-type dwarf stars in the Solar neighbourhood. In parallel, I had become involved in the projects to equip the ESO 3.6-m telescope with high-resolution spectrographs, notably the Coudé Échelle Spectrometer (CES), which was also commissioned in 1981 and greatly enhanced the quality of the stellar spectra available to the European user community. The CES enabled a group of us to undertake a detailed abundance study of a representative subsample of the stars from the uvby $\beta$ survey, which provided a comprehensive, quantitative basis for a discussion of the chemical evolution of the Galactic disk (Edvardsson et al., $A \& A$ 275, 101, 1993). The success of the CES and the development of large 2D CCD detectors led ESO to include the even more powerful spectrograph UVES in the instrumentation programme for the Very Large Telescope (VLT).

\section{Slowdown, Then a New Takeoff}

The decade of the 1990-s was a slow period for us personally, thanks to several timeconsuming administrative and managerial commitments in Denmark and abroad. Moreover, in 1995 and out of the blue, the first exoplanet was found orbiting the G star 51 Peg by our main collaborator, Michel Mayor, and his colleagues in Geneva - an epochal scientific breakthrough and a great and most well-deserved success for them. But from then on, we had to struggle on almost single-handedly with this vast body of radial-velocity data, obtained on $\sim 1,000$ nights, that demanded to be verified, collated with the photometry, calibrated and analysed. Meanwhile, HIPPARCOS had been launched, promising parallax and proper-motion data of unprecedented quantity and quality - eventually... The task appeared insurmountable in the circumstances of that time.

And of course the rest of the world was not sitting around waiting for us: Beers et al. had completed their objective-prism spectroscopic surveys, discovering large numbers of the extremely metal poor stars that were previously thought not to exist, and McWilliam and Norris had begun to scratch the surface of the wealth of new and surprising information hidden in their spectra, using 4-m telescopes. The excitement was elsewhere, while we were struggling to complete a programme begun many years before.

However, being involved with ESO during the VLT project had exposed us to its new capabilities. So, jointly with a group of European colleagues led by Roger Cayrel, we devised the "First Stars" project to chart the chemical evolution of this newly discovered population of extremely metal-poor (EMP) stars. This was basically conceived as a parallel to Edvardsson et al. (1993), based on UVES/VLT spectra and also analysed in a homogeneous manner with state-of-the-art techniques. In preparation, we spent a few years in screening several hundred candidate stars at intermediate resolution to define the most interesting and representative sample of stars to observe. But when UVES was commissioned, we were rewarded with 40 nights of VLT time, leading to a continuing series of papers labelled the "Gold Standard" by one of our competitors(!).

And finally, in 2004 and with the indispensable cooperation of Johan Holmberg, we managed to finish what is now known as the "Geneva-Copenhagen Survey of the Solar Neighbourhood" (GCS; Nordström et al., it A\&A 418, 989). Would it drown in the rising tide of huge new data sets, spearheaded by the SDSS, from much larger teams with far more advanced instruments? We feared the worst, but apparently prematurely, judging 
by the citation statistics and the many references to galactic evolution models based on the GCS, which we heard at this meeting.

And here this short, biased whirlwind tour of galactic history ends for now.

\section{Current Status of Galactic Astronomy}

Of course, technology has progressed enormously over these forty years: CCD detectors have totally replaced the photographic plate, bringing not only a huge gain in quantum efficiency and photometric precision but, in retrospect, above all the ability to establish and maintain quantitative and accurate photometric calibrations over large projects and timescales of many years; the drift-scan technique of the SDSS is the prime example. Similar improvements in IR detectors have brought us the 2MASS and similar photometric surveys and the dramatic new insights from Spitzer and Herschel, and at least some imaging capability has become available in (sub)mm telescopes, with ALMA on the horizon. HIPPARCOS revolutionised the quality and quantity of astrometry, and Gaia is poised to make another giant leap in both respects.

Efficient échelle spectrographs matched to large CCDs and fed by (multiple) optical fibres have revolutionised the speed and precision with which high-quality spectra can be mass-produced. And all this matched by similar progress in data reduction and analysis software and numerical simulations on computers with a processing power that was unimaginable 40 years ago. One wonders what the next decade may bring!

\section{Highlights of IAU Symposium 298}

Maintaining the personal perspective of this summary, my thoughts go back to IAU Symposium 254, which Joss Bland-Hawthorn, Birgitta Nordström and I organised in 2008 at the occasion of the 100th anniversary of the birth of Bengt Strömgren. With IAUS 254 we tried to organise a meeting that he himself would have enjoyed, sitting in his usual seat in the middle of the front row and excited by all the new results, without being bored by reviews of long-past achievements. I am sure that he would also greatly have enjoyed this week's symposium for the same reasons, and I have tried to imagine what his reactions could have been, now that it is over.

So what might Bengt Strömgren have remembered the best and liked the most from many talks and posters of this Symposium? On the observational front:

- The increasingly realistic simulations of $\Lambda \mathrm{CDM}$ cosmology and galaxy formation;

- The SDSS maps of the halo, with the fascinating multiple stellar debris streams and newly discovered dwarf galaxies;

- The great wealth of accurately calibrated stellar photometry;

- The great wealth and exquisite quality of modern spectroscopic data;

- The great progress in stellar physics of relevance to Galactic evolution: 3D NLTE model atmospheres; accurate stellar ages; 3D simulations of SN explosions; SN and AGB yields from new models; and the detail insights from asteroseismic analyses;

- The accuracy, volume and range of distance and proper motion data from HIPPARCOS and even more from the impending Gaia mission, complemented by radial velocities from Gaia itself and from LAMOST; but

- Above all, the much heightened attention to quality control and verification of the observational data. 
Among specific new scientific results, I speculate that he would have singled out:

- The rapidly growing number and increasingly detailed information on the dSph galaxies;

- The emerging history of the extremely metal poor halo before or beyond the metallicity range of globular clusters;

- The newly discovered multiple stellar generations with exotic chemical compositions in many "standard" globular clusters;

- The increasingly detailed knowledge of the structure of gas and dust in the ISM;

- The advanced state of chemodynamical models of the evolution of the Milky Way;

- The quantitative simulation of observational selection effects when theoretical models are compared with actual data;

- The much-improved structural parameters for the Galactic disk(s);

- The coordinated, parallel progress of theory and observations; and

- The eruption of Chinese astronomy on the international scene.

In this last context, I recall that Bengt Strömgren was first General Secretary, later President of the IAU during the peak of the cold war, while his friend and collaborator Adriaan Blaauw negotiated the re-entry of China in the IAU in 1979. He would surely have been delighted to see the achievements of LAMOST and the vigorous young research groups associated with it.

\section{What Still Needs to be Done?}

Bengt Strömgren was a polite, but fundamentally also an impatient person. So, although he would have cloaked his impatience in diplomatic terms, I am sure that he would also have pointed out the gaps in our knowledge and made comments or asked penetrating questions, like the following:

- We still don't seem to know precisely what or where the spiral arms are;

- We still don't know the precise properties and origin of 'his' "intermediate Population II", aka the "The Thick Disk";

- Why are we being so slow and inefficient in organising the radial-velocity surveys needed to match the astrometric data from space?

- Why do we still not know the nature of the Galactic bulge?

- Why do we still not know the star formation history (or histories) of the Milky Way?

- And - at the practical level - why do we still build and operate medium-size telescopes as purely national tools, when the rest of astronomy is so fully international and computer networks so transparent?

We know the methods and tools needed to deal with these issues, but also that considerable time and effort lies ahead before we can give satisfactory answers. Let us try to imagine what could be done over the next ten years.

\section{Looking A Decade Ahead To 2023}

Given the progress and preparations we have heard about this week, I believe we can have reasonable confidence in expecting the following achievements to happen by $\sim 2023$ :

- The fully reduced full-mission data set from Gaia;

- The spectroscopic follow-up surveys to Gaia well under way;

- The SDSS/Apogee surveys completed (perhaps also in the south?); 
- ALMA fully complete and a submm survey telescope under way;

- LOFAR in full operation, Phase I of the SKA well under way;

- Star formation in the Galaxy "well understood", based on better models and the combined data sets from Spitzer, Herschel and ALMA;

- Accurate chemical yields being derived from 3D models of SN explosions and of nucleosynthesis in the envelopes of AGB stars;

- Complete grids of 3D NLTE model atmospheres for most stellar mass ranges; and

- Convergence of the competing (chemo)dynamical models of the Milky Way.

On the promise of this week's meeting, we should be enjoying an exciting time at IAU Symposium $\sim 375$ around the year 2023. See you all there!

Acknowledgements: My first and greatest debt is to my lifelong collaborator Birgitta Nordström for sharing this forty-year scientific journey, and to the SOC and LOC for dedicating this meeting to both of us and making it such a memorable experience in all respects - including an unforgettable conference/birthday banquet. Our own research projects have been made possible by large allocations of observing time, primarily at ESO and Danish telescopes in Chile, and have been shared with far too many international collaborators to name them all here; it was a pleasure and an honour to see many of them again in Lijiang. Generous financial support, throughout the years notably from the Danish Natural Science Research Council and the Carlsberg Foundation over many years, and specifically by the LOC of this meeting, is gratefully acknowledged. 